\title{
SUPPORTING SITUATION AWARENESS USING NEURAL NETWORK AND EXPERT SYSTEM
}

\author{
MOHSEN NADERPOUR*, JIE LU \\ Decision Systems and e-Service Intelligence Laboratory \\ Centre for Quantum Computation and Intelligent Systems (QCIS) \\ School of Software, Faculty of Engineering and Information Technology \\ University of Technology, Sydney, P.O. Box 123, Broadway, NSW 2007, Australia
}

\begin{abstract}
Situation awareness (SA) is a critical factor for human decision making and performance in dynamic environments. Actually SA is a mental model of the current state of the environment and includes many types of complex systems such as safety supervisory systems. The current paper employs two focus areas including neural network and expert system for maintaining SA in a safety supervisory system. The neural network components provide adaptive mechanisms for perception, and the expert system offers the ability to support comprehension and projection.
\end{abstract}

\section{Introduction}

Situation awareness (SA) involves being aware of what is happening in the vicinity to understand how information, events, and actions will impact the goals and objectives. Lacking or inadequate SA has been identified as one of the primary factors in accidents attributed to human error. Thus, SA is especially important in work domains where the information flow can be quite high, and poor decisions may lead to serious consequences. The idea did not receive much attention until the late 1980s, but has become a hot topic ever since.

The primary research came from the aviation industry but a similar review in other domains, such as nuclear power showed that this is not a problem limited to aviation, but one faced by many complex systems where combining and presenting vast amounts of data now available from many technological systems in order to provide true SA is a big challenge (whether it is to a pilot, a physician, a business manager, or an automobile driver) [1].

Despite having its roots in aviation, it has been suggested that the concept is equally applicable to human supervisory control for land based industries. It is also argued that problems in human supervisory control may be due to poor situational awareness [2], such as: 1) failure to detect critical cues regarding the

\footnotetext{
**ahsen.Naderpour@student.uts.edu.au
} 
state of the system; 2) failure to interpret the meaning of information perceived via SCADA (supervisory control and data acquisition) technology; 3) failure to understand individual task responsibilities and the responsibilities of others; 4) failure to communicate with other operators in the team; and 5) failure to communicate with other teams.

This paper considers the applicability of SA concepts to safety in the control of complex systems. Safety supervisory systems continue to increase in degree of automation and complexity as operators are decreasing. As a result, each operator must be able to comprehend and respond to an ever increasing amount of available risky status and alert information. In our previous work, we proposed an innovative conceptual model that assesses situations and helps the decision maker to take appropriate action in hazardous situations [3]. In this paper, we will describe how neural network and expert system can support SA in a complex environment.

\section{Basic Concepts}

\subsection{Situation Awareness}

One of the earliest and most widely used definitions of SA describes it as the "perception of the elements in the environment within a volume of time and space, the comprehension of their meaning and the projection of their status in the near future". Based on this definition, SA is comprised of three levels: (1) perception, (2) comprehension, and (3) projection.

Level 1 SA, perception, involves the sensory detection of significant environmental cues. For example, operators need to be able to see relevant displays or hear an alarm sound. Comprehending the meaning or significance of that information in relation to operator's goals is also important. Operators with good Level 2 SA are able to understand the immediate impact of an outage on other parts of the system. Projection, the highest level of SA, consists of extrapolating information forward in time to determine how it will affect future states of the operating environment [1].

\subsection{Neural Networks}

Neural network or artificial neural network (ANN) is the interconnection of artificial neurons that tends to simulate the nervous system of a human brain. ANNs are commonly used in machine learning and utilize supervised, unsupervised, and reinforcement learning approaches to achieve predictive properties based on example data. Unsupervised learning can be effective when 
ground-truth is not available with a dataset. Supervised learning provides a means to use experience to correctly classify yet unseen situations. Reinforcement learning offers promise for machine learning in difficult learning environments by taking advantage of feedback about the performance of a system. In particular, they can be embedded into appropriate sensors for operation at the lowest levels of information fusion with effective, but lowcomplexity designs. Although neural networks have been applied to sensor fusion, their use in SA has been limited, possibly because of the lack of rich training data for this problem [4].

\subsection{Expert Systems}

The basic idea behind expert systems (ES) is simply that expertise, which is the vast body of task-specific knowledge, is transferred from a human to a computer. This knowledge is then stored in the computer and users call upon the computer for specific advice as needed. The computer can make inferences and arrive at a specific conclusion. Then like a human consultant, it gives advices and explains, if necessary, the logic behind the advice. A rule-based ES is defined as one, which contains information obtained from a human expert, and represents that information in the form of rules, such as IF-THEN. The rule can then be used to perform operations on data to inference in order to reach appropriate conclusion. These inferences are essentially a computer program that provides a methodology for reasoning about information in the rule base or knowledge base, and for formulating conclusions [5].

\section{Supporting Situation Awareness Using NN and ES}

In an attempt to effectively provide SA for a decision maker, we have brought together two focus areas of research. An overview of these areas is depicted in Figure 1. For determining the aspects that are important for an operator's SA in a safety supervisory system, we use the goal-directed task analysis which was introduced in [1]. The results are showed in Table 1.

In a complex and dynamic environment, a casualty requires immediate response. There are often multiple actions required in a very short time and the trend toward smaller operators' size and increasing automation has resulted larger volumes of more and more complex information in a dynamic environment. Therefore, it is necessary to assist the operators in quickly understanding the available information by providing that data in a format that allows them to act quickly and correctly. The information provided for situational awareness must be more than just information gathering. This implies 
collecting the right multi-domain information across a net-centric environment for shared awareness and presenting results for the human to understand and make quick decisions. Any new approach must efficiently bring together the human operator, sensor equipment data, and real world events to provide a subset of actionable information [4].

Table 1. Safety goals and decisions

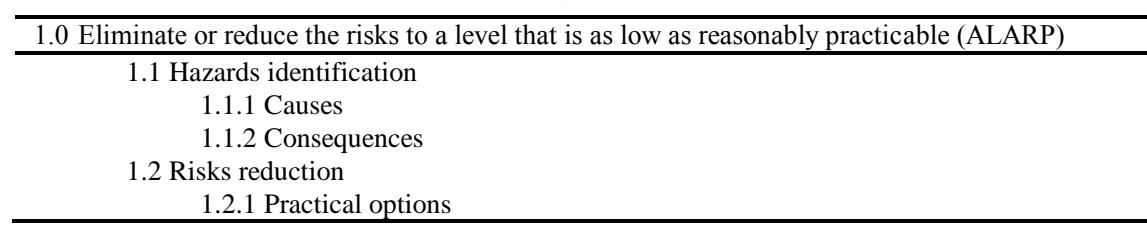

For the safety supervisory system, critical factors in the environment (SA level 1) relate to the actual state of the system and identifying the hazards. In this step we rely on neural networks. The historical database that includes information related with normal and abnormal situations can be used to train the ANN structure. The on-line or off-line measurements from the plant are the ANN's inputs. The ANN's outputs are the signals of different suspected hazards. These outputs are a subset of the set of ES's inputs.

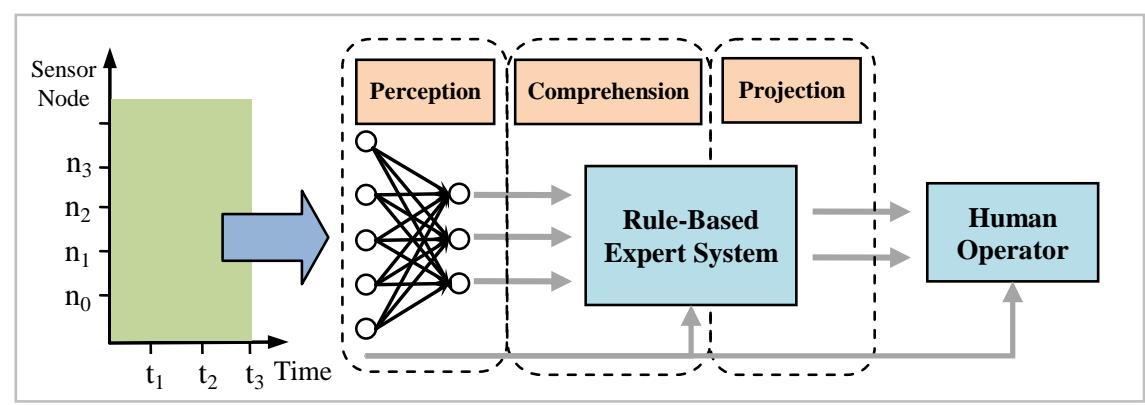

Figure 1. Situation awareness diagram

SA level 2 relates to the operator's understanding of the system as a whole, and emergent events. At this level, it is also necessary to understand the causality and consequences of the hazards. For SA level 3, the system and operator should understand future required actions. This is not only limited to responding to changing engineering conditions, it also addresses the changing tactical situation to eliminate or reduce the risks. For both these levels we utilize an expert system. The HAZOP analysis and FTA are useful to build the knowledge base (KB). This base relies on the knowledge of the operators and engineers about the process and allows formulating artificial intelligence algorithms. Our approach 
still relies on the effectiveness of the operator. If the approach is focused solely on the automated features, then the operator can become more disconnected from the tools and resources needed to assess situations and make objective and effective decisions.

\section{Functional Demonstration}

Chemical plants are one of the complex environments that the supervision tasks have increased considerably. A decision support system is needed to assist operators in understanding and assessing status and responding quickly to risky situation. For example, in the explosion of Texas City, TX BP Amoco Refinery, that 15 workers were killed and 170 injured, the key problem was the poor operator's SA in a complex, fast moving environment [6]. For functional demonstration, an ethylbenzene process plant, involving two reactors and two distillation columns, is chosen. The safety system includes eleven sensors which describe in Table 2. For perception phase we use BP neural network that has eleven inputs $\left(\mathrm{I}_{1}, \ldots, \mathrm{I}_{11}\right)$ and 6 outputs $\left(\mathrm{O}_{1}, \ldots, \mathrm{O}_{6}\right)$ that show the possible hazards for this plant as shown in Table 2. Using steady state data from simulation or from experimental data, when available, the ANN is trained and the outputs are useful for the next step. The activation functions are sigmoidals in the hidden layer and linear in the output.

Table 2. Sensors and hazards

\begin{tabular}{ccccc}
\hline Zone & Sensor & Description & Hazard & Description \\
\hline \multirow{2}{*}{ R1 } & TCR1 & Temperature controller & H1 & High Temperature in R1 \\
& LCR1 & Level controller & H2 & High Temperature in R2 \\
\hline R2 & LCR2 & Level controller & H3 & High Pressure in R1 \\
& TCD1 & Temperature controller & H4 & High Level in D1 \\
D1 & PCD1 & Pressure controller & H5 & High Level in D2 \\
& LC1D1 & Level controller & H6 & High Pressure in D2 \\
& LC2D1 & Level controller & & \\
& TCD2 & Temperature controller & & \\
& PCD2 & Pressure controller & & \\
D2 & PC1D2 & Level controller & & \\
& L & & \\
\hline & LC2D2 & Level controller & & \\
\hline
\end{tabular}

For comprehension and projection phases, a set of if-then rules has been defined to build the knowledge base of expert system. The antecedents are usually the outputs of the ANN with or without some measured/calculated variables. For example the rule which is relevant to $\mathrm{H} 1$, is shown in Table 3. To 
simplify and accelerate the performance of expert system, forward reasoning that starts from the fact, reasoning towards target is used.

Table 3. Set of rules

\begin{tabular}{ccccccc}
\hline \multicolumn{2}{c}{ Rule } & Antecedent & Causes & Consequences & Suggestions \\
\hline Rule 1 & IF & H1 & & & \\
\hline & THEN & & $\begin{array}{l}\text { Poor cooling or } \\
\text { input low level }\end{array}$ & $\begin{array}{l}\text { shut-down, release } \\
\text { and explosion }\end{array}$ & $\begin{array}{l}\text { switch to redundancy pump in } \\
\text { cooling system and } \\
\text { administrative checks }\end{array}$ \\
\hline
\end{tabular}

\section{Conclusion and Future Work}

As safety supervisory systems continue to increase in degree of automation and complexity, the task of providing actionable information for SA becomes more difficult and costly to achieve. The proposed approach employs neural network and expert system to support SA in a safety supervisory system. Reinforcement learning is a general area of research worth pursuing in the area of SA, so in future work we will attempt to create principles for employing reinforcement learning to support SA.

\section{References}

1- M. R. Endsley, "Situation Awareness," in Handbook of Human Factors and Ergonomics, G. Salvendy, Ed.: John Wiley and Sons Press 2006, pp. 528542.

2- N. A. Stanton, P. R. G. Chambers, and J. Piggott, "Situational awareness and safety," Safety Science, vol. 39, pp. 189-204, 2001.

3- M. Naderpour, J. Lu, and E. Kerre, "A Conceptual Model for Risk-Based Situation Awareness," in International Conference on Intelligent Systems and Knowledge Engineering (ISKE), Shanghai, China, 2011, pp. 297-306.

4- N. G. Brannon, J. E. Seiffertt, T. J. Draelos, and D. C. Wunsch Ii, "Coordinated machine learning and decision support for situation awareness," Neural Networks, vol. 22, pp. 316-325, 2009.

5- L. Shu-Hsien, "Expert system methodologies and applications: a decade review from 1995 to 2004," Expert Systems with Applications, vol. 28, pp. 93-103, 2005.

6- J. L. Pridmore, "Designing for the improvement of operator situation awareness in automation systems," Ph.D. Thesis, United States-Alabama: Auburn University, 2007. 